\title{
El derecho a una muerte digna en Argentina: la judicialización de la toma de decisiones médicas en el final de la vida
}

\section{| 1 Juan Pedro Alonso |}

Resumen: El artículo discute desde una perspectiva socio-histórica el contexto y las implicancias de los procesos de judicialización en torno a la gestión médica de la muerte y el morir. El objetivo del artículo es describir y analizar la judicialización de la toma de decisiones médicas en el final de la vida en Argentina, a partir de fallos judiciales producidos entre 1975 y 2015. Se examina el contexto y las principales características de la litigación respecto a estas decisiones, así como los cambios producidos en el periodo: los casos que son objeto de disputa (desde el rechazo de transfusiones al pedido de retiro de medidas de soporte vital), los argumentos y leguajes utilizados (de la objeción de conciencia a la autonomía) y la emergencia de nuevos derechos. Por último se discuten las consecuencias y las perspectivas futuras de la judicialización en este campo.

> Palabras-clave: judicialización; medicalización; muerte digna, bioética, Argentina.
1 Instituto de Investigaciones Gino Germani. Area Salud y Población. Buenos Aires, Argentina (juanpedroalonso79@ gmail.com).

Recibido: 24/07/2015 Aprobado: 15/12/2015 


\section{Introducción}

Los procesos de judicialización remiten a la ampliación de la esfera de competencia e intervención por parte de cortes y tribunales acerca de diferentes áreas de la vida social y política, anteriormente resueltas en otros ámbitos públicos o en forma privada (CARVALHO, 2004; SIEDER et al., 2008; VENTURA, 2010; ASENSI, 2010; ABDO-FEREZ, 2014). Asimismo, estos procesos refieren a la adopción, por parte de individuos y grupos, de un discurso basado en derechos como vía para instrumentar demandas o reclamos privados y/o colectivos, descripto también en términos de movilización legal (SMULOVITZ, 2008, 2008a; CUENCA; PICCONE, 2011; DELAMATA, 2013). Los procesos de judicialización puede adquirir diferentes formas y sentidos de acuerdo a los actores que la movilizan o protagonizan (los mismos jueces, actores políticos, activistas, ciudadanos), y a los objetivos a los que apuntan estas estrategias.

La creciente literatura sobre la judicialización y la movilización legal en América Latina ha abordado procesos de judicialización en diferentes temáticas y contextos, como los reclamos sobre terapias de cambio de sexo en personas trans en Brasil y Argentina (VENTURA, 2010; FARJI-NEER, 2012), conflictos previsionales y demandas de ahorristas en Argentina (SMULOVITZ, 2008, 2008a), en temas de violencia doméstica (MACAULAY, 2008) y reclamos por el derecho de pueblos originarios (RAMÍREZ, 2014), entre muchos otros. En el campo de la salud, el análisis de los procesos de judicialización en el ámbito local y regional se han centrado en disputas por el derecho a la salud, en virtud de demandas por el acceso o la ampliación en la cobertura de medicamentos y servicios (MOTTA FERRAZ, 2009; VENTURA et al., 2010; BERGALLO, 2013; YAMÍN; GLOPPEN, 2013; DINIZ, 2014, entre otros), y en menor medida en el impacto de la judicialización de la práctica y las decisiones médicas, como los juicios de mala praxis profesional y su impacto en la configuración de lo que se ha denominado como "medicina defensiva" (GHERARDI; GHERARDI, 2007).

El presente artículo se inscribe en parte en esta última línea de trabajos, orientado ya no sólo a examinar el impacto de la litigación en la práctica médica sino a describir desde una perspectiva sociológica el contexto y las implicancias de los procesos de judicialización en torno a la gestión médica de la muerte y el morir. Puntualmente, el objetivo del artículo es describir y analizar la judicialización de la toma de decisiones médicas en el final de la vida en Argentina en las últimas 
décadas. Esto es, el recurso a tribunales y jueces para demandar la realización o rechazo de intervenciones médicas en las que se pone en juego el fin de la vida, realizados por pacientes y/o familiares o por profesionales o instituciones de salud: desde pedidos que apuntan en forma directa a facilitar o entorpecer que una muerte inminente ocurra (como el retiro o continuidad de medidas de soporte vital) a situaciones en que el rechazo de un tratamiento supone un peligro de muerte más o menos inminente (como el rechazo de intervenciones de pacientes con enfermedades avanzadas o graves, o que sea el rechazo de intervenciones lo que coloque a los pacientes en estado crítico).

El artículo se enmarca en una investigación en curso que aborda desde una perspectiva socio-histórica los debates y discursos públicos en torno a la gestión médica del final de la vida en Argentina, examinando el tratamiento de la temática en diferentes arenas de discusión (legislativas, mediáticas y científicas, entre otras). ${ }^{1}$ Interesa aquí analizar la presencia y las características de estos debates en la arena jurídica, a partir del análisis de un cuerpo de fallos producidos por tribunales argentinos (a nivel nacional, provincial y local) entre los años 1975 y 2015, periodo que comprende desde los primeros casos de judicialización de la temática a nivel mundial a un fallo reciente del máximo tribunal de justicia del país que avala la constitucionalidad de la ley de "muerte digna" sancionada en el año 2012. ${ }^{2}$

A partir de la segunda mitad del siglo XX una serie de innovaciones técnicas (como la ventilación pulmonar, las técnicas de resucitación y la nutrición artificial, entre otras) dieron pie a la emergencia de la terapia intensiva, posibilitando la prolongación artificial de la vida (MENEZES, 2000; GHERARDI, 2007). En el marco de estos avances en la medicalización y tecnificación del final de la vida, que entre otras cuestiones derivó en el cambio en los criterios para determinar la muerte (LOCK, 2002), en la década de 1970 comenzaron a cobrar visibilidad casos controversiales en la toma de decisiones en el final de la vida, como el de Karen Quinlan, que marcó un hito en la consideración pública mundial sobre estos temas. El movimiento de los hospices y los cuidados paliativos (CLARK; SEYMOUR, 1999; CASTRA, 2003; MENEZES, 2004); la emergencia de la bioética y el énfasis en la autonomía de los pacientes (ROTHMAN, 1991); y los movimientos a favor de la legalización de la eutanasia y del derecho a morir con dignidad (McIERENEY, 2000), que estos casos contribuyeron a vitalizar, pusieron en el centro del debate público el modo en que el morir y la muerte 
eran gestionados en ámbitos médicos, así como a el derecho de los pacientes a participar en la toma de decisiones en los momentos finales. Casos como los de Nancy Cruzan y Terri Schiavo en los Estados Unidos, por nombrar sólo algunos, continuaron y continúan avivando el debate público sobre la eutanasia y la muerte digna. Asimismo, como lo evidencian las batallas legales que estos casos suscitaron, dan cuenta del lugar que la arena judicial tiene en la tramitación de los conflictos en torno a estas temáticas.

Previamente a la sanción de la Ley 26.742, más conocida como ley de "muerte digna”, no existía en Argentina un marco legal específico que amparara las decisiones de abstención o suspensión de tratamientos en el final de la vida. Si bien en el marco normativo argentino el derecho a rechazar tratamientos estaba contemplado en la ley 17.132 de Ejercicio de la medicina, vigente desde 1967, que obligaba a los profesionales de la salud a respetar la voluntad de los pacientes cuando se negaran a realizar un tratamiento, ${ }^{3}$ el reciente proceso de discusión parlamentaria de una ley dedicada a la temática evidenció la incertidumbre y el vacío legal que reinaban respecto a quiénes y en qué condiciones podían rechazar tratamientos que mantuvieran con vida a una persona. La ley 26.529 de Derechos de los pacientes, del año 2009, contemplaba el derecho a rechazar tratamientos y procedimientos médicos y regulaba las directivas anticipadas, pero fue la modificación sancionada el año 2012 la que explicitó las condiciones y los tratamientos pasibles de ser rechazados en el final de la vida.

A continuación se describen las transformaciones ocurridas en la judicialización de la toma de decisiones en el final de la vida en Argentina, observando los cambios en los tipos de demandas, en los argumentos y lenguajes, así como en la formulación de nuevos derechos. Por último se realiza un balance de la judicialización de la temática, examinando aspectos negativos y positivos de estos procesos; la pregnancia del discurso de los derechos en los planteos relativos al cuerpo y la salud; y las perspectivas futuras de judicialización en este campo.

\section{La "muerte digna” en los tribunales argentinos}

¿Qué tipos de casos llevan al ámbito jurídico la toma de decisiones en el final de la vida? ¿Quiénes presentan estas demandas y qué respuesta encuentran en los tribunales? ¿Cómo evolucionan las demandas y en los fundamentos en que se basan las sentencias? ¿Cuándo emerge el derecho a la "muerte digna" 
en la jurisprudencia? En primer lugar se describen el desarrollo del proceso de judicialización de la toma de decisiones en el final de la vida, atendiendo a los quiebres que se producen en el tipo de casos que llegan a la justicia, los lenguajes y argumentos con que se aborda el tema, y en la emergencia y consolidación del derecho a una "muerte digna" para luego discutir los alcances y perspectivas de la judicialización de estas decisiones.

\section{Los primeros casos: el rechazo a recibir transfusiones sanguineas}

La judicialización de la toma de decisiones relativas al final de la vida comienza a girar en torno a conflictos derivados del rechazo de trasfusiones sanguíneas por motivos religiosos. Por su negativa a recibir transfusiones sanguíneas, basada un pasaje bíblico, los Testigos de Jehová han generado una importante jurisprudencia sobre el derecho a rechazar tratamientos en muchos países.

El primer caso en llegar a los tribunales argentinos y obtener sentencia se produce en 1975 (A. de L., S. E. s/amparo, 13 de agosto 1975). El padre de una mujer que necesitaba una transfusión por una complicación postparto recurre a la justicia para que autorice la intervención, a la que se oponía el marido porque ambos eran Testigos de Jehová. El juzgado hace lugar al pedido del padre y fuerza a la institución de salud a realizar el tratamiento médico, basando la sentencia en la protección del valor de la vida como bien supremo. La transfusión de sangre se consideraba un tratamiento seguro y debía primar el derecho de los médicos a cumplir con sus obligaciones: proteger la vida del paciente aún contra su voluntad y/o la de su familia.

A mediados de la década de 1980 se registraron varios casos de pedidos de autorización para realizar transfusiones en forma compulsiva por parte de instituciones de salud y de familiares de pacientes, y comienza a variar la jurisprudencia sobre esta temática. En algunos de estos casos la decisión afectaba el interés de menores de edad, como el caso de los padres que no aceptaban la realización de una transfusión de sangre para su hija recién nacida (A.Q., J.R., 11 de noviembre de 1986). ${ }^{4}$ En esos años un tribunal rechazó el pedido de los hijos de un paciente que se negaba a recibir transfusiones (R., N. E., 4 de diciembre de 1986). En el fallo se consideraba el derecho a la vida, a la integridad personal y a la salud como bienes de interés público, pero sobreponía a ellos el derecho a la dignidad personal: 

convicciones religiosas, que pueden llevar inclusive -como en este caso- a la muerte. Por ello, deniego lo pedido. (R., N. E., 4 de diciembre de 1986).

El fallo es el primero en reconocer el derecho de los pacientes a rechazar tratamientos, avalando la objeción de conciencia por motivos religiosos.

\section{Rechazo de tratamientos y postura de la Corte Suprema de Justicia}

En la década de 1990 el tema adquiere mayor presencia en los tribunales, tal vez alentado por la amplia difusión de casos internacionales que mantienen los debates sobre la eutanasia en la agenda pública y por modificaciones constitucionales que facilitaron el acceso a los tribunales. ${ }^{5}$ Durante esta década comienzan a llegar a la justicia demandas relacionadas con otro tipo de casos, y se producen los primeros pronunciamientos de la Corte Suprema de Justicia de la Nación en relación a estas temáticas.

Si bien en el aumento en la judicialización de las decisiones médicas en el final de la vida continuó primando la disputa en torno a trasfusiones sanguíneas, durante estos años aparecen fallos en los que se discute el derecho a rechazar tratamientos por otras razones que la adscripción religiosa. El primero de estos fallos es de 1991, en el que un juzgado de Mar del Plata resuelve rechazar el pedido del hijo de un paciente para que se le realice en forma compulsiva la amputación de una pierna (J., J., 21 de febrero de 1991). El pedido del hijo se basaba en la supuesta falta de competencia del paciente para tomar esa decisión, y en el hecho de que su negativa supondría un desenlace fatal. La sentencia avaló el rechazo de la intervención por parte del paciente, en virtud de derechos relativos al propio cuerpo y a la integridad física, y ya no amparándose en la libertad religiosa y la objeción de conciencia. El fallo inaugura así una línea argumentativa que se afianzará en la jurisprudencia posterior y que pone en el centro de la toma de decisiones al propio paciente, en consonancia con los principios de la bioética y con la doctrina del consentimiento informado:

No surgiendo que la conducta del paciente que no consiente que se le realice una urgente intervención quirúrgica, configure una forma de suicidio, debe respetarse la voluntad de aquél y la solución viene impuesta por la naturaleza de los derechos en juego que determinan que el paciente sea el árbitro único e irremplazable de la situación. El principio expuesto no debe ceder aunque medie amenaza a la vida. (J., J., 21 de febrero de 1991). 
En estos años también se pronuncia por primera vez la Corte Suprema de Justicia de la Nación respecto a estas temáticas. En un fallo del año 1993, la Corte avala el pedido de un paciente que se niega a recibir una transfusión de sangre por motivos religiosos (Bahamondez, Marcelo s/ medida cautelar, 6 de abril de 1993). A causa de una hemorragia digestiva los profesionales que lo atendían propusieron realizar una transfusión sanguínea, y para proteger sus derechos recurrió a la justicia. En primera y segunda instancia el pedido fue rechazado, enmarcado como un intento de "suicidio lentificado", por lo que el defensor oficial del paciente presentó un recurso extraordinario ante la Corte Suprema, que dejó sin efectos las sentencias previas y reconoció el derecho a rechazar la intervención. La importancia del fallo reside en la opinión de varios de los integrantes del máximo tribunal, que colocan la decisión de rechazar tratamientos o intervenciones en el marco de las libertades individuales (a la dignidad, a la autonomía, a decidir sobre la propia vida y el cuerpo) y desestiman que por tales decisiones se vea afectado un interés social o el derecho de terceros. Asimismo, el fallo ilustra el pasaje de justificar la negativa a recibir determinadas intervenciones médicas en virtud de la libertad religiosa, a la afirmación de derechos personalísimos, para ejercer los cuales la condición religiosa no es relevante (voto de los jueces Belluscio y Petracci, Bahamondez, Marcelo s/ medida cautelar, 6 de abril de 1993).

\section{Nuevos argumentos y lenguajes: bioética y derechos humanos}

El fallo de la Corte marca un quiebre en la argumentación (y dirección, aunque con excepciones) de las sentencias frente a casos de rechazo de tratamientos. En los años siguientes se encuentran nuevos lenguajes y argumentos para fundamentar las sentencias, que sin embargo continúan girando en torno al valor central de la autonomía. Estos argumentos remiten, por un lado, a la inscripción de la toma de decisiones en el final de la vida bajo el paraguas de los derechos humanos; y por otro a la incorporación de principios provenientes de la bioética, que comienza a afirmarse como un discurso relevante en este campo. Varios autores hacen referencias al "bioderecho" para dar cuenta de las relaciones entre estas disciplinas, movilizadas por los debates que suscitan las nuevas tecnologías médicas y los avances en las ciencias de la vida (HOOFT, 2002; HOOFT; PICARDI, 2006; DINIZ, 2003, 2006; VENTURA, 2010). Estos cruces supondrán tanto la 
participación de bioeticistas o de comités de bioética como expertos consultados por los jueces para informar sus decisiones (ver DINIZ, 2006), o en la adopción del lenguaje de esta disciplina en la fundamentación de los fallos.

Un fallo del juez marplatense Pedro Hooft ilustra estas variaciones en los argumentos desplegados en las sentencias (P.A., F., 18 de septiembre de 1995). Una institución de salud pide autorización para amputar a un paciente diabético que se niega a ser sometido a dicha operación, pedido que es rechazado por el tribunal en virtud de la legislación (un artículo de la referida Ley 17.132 de Ejercicio de la medicina que obliga a los profesionales a solicitar la conformidad del paciente para operaciones mutilantes) y de normas constitucionales que protegen las libertades individuales. El fallo evidencia estos cruces entre bioética y derecho que, en años posteriores, serán corrientes en este tipo de casos: es el primer tribunal que recurre a la opinión de un Comité de Ética y que utiliza principios de la bioética para justificar la decisión judicial. Un fragmento del fallo:

La cuestión traída a decisión, es de naturaleza Bioética, en un ámbito en el que esta novel transdisciplina se vincula estrechamente con el "bioderecho" [...] Ha constituido un aporte importante de la Bioética, el reconocimiento del paciente como agente moral autónomo (P.A., F., 18 de septiembre de 1995).

¿A qué fuentes recurren los jueces para obtener opiniones autorizadas sobre estos temas? Resulta interesante observar cómo se incorporarán paulatinamente este tipo de fuentes como relevantes para la decisión judicial. Con el paso del tiempo será primero más común y luego la regla encontrarse con otros actores y voces autorizadas, tales como expertos en bioética y/o Comités de Ética, además de los peritos e informantes médicos, que dominaban la escena hasta entonces. Por último, por primera vez en casos de este tipo el fallo introduce la referencia al "derecho del enfermo de 'morir con dignidad", sobre el que volveremos más adelante.

\section{Pedidos de retiro de soporte vital y directivas anticipadas}

A partir del año 2000 comienzan a llegar a los tribunales argentinos casos en los que se discute el retiro de medidas de soporte vital. ${ }^{6}$ Con estos casos, por sus características, comienzan a discutirse nuevos instrumentos legales, como las directivas anticipadas o testamentos vitales, en que los que está en juego es a quién le corresponde decidir cuando el paciente no puede hacerlo y en base a qué 
criterios, a la vez que la posibilidad de decidir anticipadamente los cuidados que se desean recibir (o no) en el final de la vida.

Los primeros en recurrir a la justicia para pedir el retiro de este tipo de tratamientos son los padres de una niña recién nacida, afectada por una enfermedad terminal (Benítez, M. y otros c. GCBA, 16 de noviembre de 2001). El antecedente más relevante sobre este tópico, sin embargo, es el pedido del marido de una mujer en estado vegetativo permanente, quien reclama que se le retire la hidratación y alimentación artificial que la mantiene con vida. El pedido fue rechazado en primera instancia, el marido apeló la sentencia y la Suprema Corte de Justicia de la Provincia de Buenos Aires confirmó la negativa (M. d. C., 9 de febrero de 2005). El caso es importante en el desarrollo de la judicialización de la toma de decisiones en el final de la vida por varios motivos: es uno de los primeros fallos en que se discute la validez de las directivas anticipadas como instrumentos apropiados para la toma de decisiones y en que se plantea la suspensión de medidas de soporte vital en personas en estado vegetativo, ${ }^{7}$ entidad clínica sobre los que giran actualmente los principales debates científicos y éticos en el final de la vida (CONSTABLE, 2012; NETTELTON et al., 2014; FINS, 2015).

Los argumentos que justificaron la negativa al retiro de medidas de soporte vital para algunos miembros de la Suprema Corte fueron la salvaguarda del derecho a la vida, dado que la suspensión de los cuidados era considerado una práctica eutanásica, y al desacuerdo de que un tercero (y no el propio paciente) pudiera tomar una decisión de estas características. Como señala unos de los jueces del tribunal, el derecho a morir con dignidad:

[...] es un derecho personalísimo, inherente a la persona y que, como tal, sólo puede ser ejercido por su titular. No se concibe que el mismo pueda ser ejercido por un tercero con total ignorancia de lo que podría desear o querer el titular de esa vida (Voto del juez Roncoroni, M. d. C, 9 de febrero de 2005).

Otros miembros de la Corte, por su parte, convalidaban la posibilidad de rechazar los tratamientos, dada la irreversibilidad del cuadro clínico, e incluso ponderaban la validez de las directivas o voluntades anticipadas. Pero la falta de un mandato expreso de la paciente y la disputa familiar sobre cuáles serían sus mejores intereses (los padres y los hermanos de la mujer se oponían a que se retiraran los cuidados) impedían que pudiera ser un argumento que respaldara la demanda (Voto de la jueza Kogan, M. d. C., 9 de febrero de 2005). En 
consonancia con estos argumentos, fallos posteriores reconocieron la validez legal de las directivas anticipadas, como en el caso de una mujer afectada por una enfermedad degenerativa que ante la perspectiva de la progresión de la enfermedad reclamó a la justicia que en el futuro se respetara su voluntad de "no someterse a ninguna práctica que prolongue su vida en forma artificial por medio de procedimientos médicos invasivos" (M., 25 de julio de 2005). En el mismo sentido, pero en un caso que involucraba a un menor, una institución de salud demandó autorización a la justicia para respetar la decisión de los padres de un niño con una enfermedad degenerativa para que se abstuvieran de utilizar medidas de soporte vital (E.C., 20 de marzo de 2006). ${ }^{8}$

Otro punto de disidencia entre los jueces de la Suprema Corte de la provincia de Buenos Aires fue el estatuto de las medidas de hidratación y alimentación artificial (¿una medida extraordinaria y desproporcionada en relación a las perspectivas de mejoría o cuidado ordinario?) y la posibilidad de suspenderlas; discusión que adquirirá relevancia en los debates posteriores en torno a la ley de “muerte digna” (ver ALONSO, 2014).

\section{La muerte digna como derecho}

Como hemos señalado anteriormente, el fallo del juez Hooft (P.A., F., 18 de septiembre de 1995) es el primero en enmarcar el rechazo de tratamientos como parte de un derecho (no muy claramente definido ni establecido) a "morir con dignidad". En años anteriores, el derecho a una "muerte digna" comienza a formularse a propósito de pedidos de excarcelación de personas en estadios terminales de patologías como el VIH/Sida, en momentos previos a la cronificación de la enfermedad (ver por ejemplo E.C.S, 30 de agosto de 1993). Si bien algunos fallos posteriores apelan a este "derecho" para avalar la decisión autónoma de rechazar intervenciones, no será hasta el año 2012, cuando el Congreso de la Nación sancione la Ley 26.742 que modifica la Ley de Derechos de los Pacientes, que la "muerte digna" adquiera un estatuto legal en la Argentina (Alonso et al 2014). La ley garantiza el derecho de los pacientes con enfermedades terminales o irreversibles (o de familiares en su nombre) a rechazar terapias o procedimientos médicos cuando estos sean desproporcionados a las perspectivas de mejoría o cuando prolonguen la agonía, incluidas las medidas de soporte vital como la hidratación y la alimentación artificial, y reconoce a su vez la validez de las directivas anticipadas. Un fallo reciente de la Corte Suprema de Justicia de 
la Nación confirmó en el año 2015 la constitucionalidad del emergente derecho

a "morir con dignidad", al autorizar el retiro de soporte vital de un paciente que llevaba más de 20 años en un estado de conciencia mínima (D., M.A s. declaración de incapacidad, 7 de julio de 2015).

Como lo evidencia este caso, la existencia de esta nueva norma no supone la interrupción de la judicialización de la toma de decisiones en el final de la vida, punto al que la redacción de la ley aspiraba. En el marco de la aprobación de la ley de "muerte digna" la familia de un paciente que llevaba 15 años en estado vegetativo apeló un fallo previo a la sanción de la ley, que rechazaba el pedido de retiro de soporte vital. El Tribunal Superior de Justicia de Neuquén, que recibió la apelación, sostuvo que el pedido de la familia se encontraba enmarcado por los lineamientos de la nueva ley, pero la apelación del defensor oficial del paciente (que cuestionaba que existiera una voluntad clara del paciente así como la legalidad de la suspensión de la hidratación y la alimentación, que consideraba eutanásicas) y llevó el caso a la Corte Suprema de Justicia de la Nación. Ante la falta de un documento formal, la Corte se basó en las declaraciones de las hermanas, que manifestaron la voluntad del paciente de no ser mantenido con vida en esas situaciones. Una de las hermanas refirió la oportunidad en que junto a su hermano leían acerca del caso de Karen Quinlan, primero en poner el tema en la agenda pública, y que en un momento "su hermano M. A. D. la miró y le dijo 'si alguna vez a mí me pasa algo, a mí me dejás morir”" (citado en D. M. A., 19 de abril de 2013).

\section{La judicialización de la toma de decisiones en el final de la vida: balance y perspectivas}

¿Cuál es el balance de la judicialización de la toma de decisiones en el final de la vida en Argentina? ¿Ha promovido el desarrollo de una medicina defensiva o el reconocimiento de la autonomía de los pacientes y la salvaguarda de sus derechos? ¿Qué lugar ha tenido el discurso de los derechos en las reivindicaciones de pacientes y familiares en torno a planteos relativos al cuerpo y la salud en el final de la vida? ¿Cuáles son los posibles avatares de la judicialización en este ámbito?

En líneas generales los trabajos que analizan el avance de la judicialización en el campo de la salud señalan las consecuencias negativas de dichos procesos. Por el predominio de la litigación individual sobre la colectiva y la desigualdad en el 
acceso a la justicia, entre otros aspectos, la judicialización en torno al derecho a la salud contribuiría a profundizar la inequidad al interior de los sistemas sanitarios y en el acceso a bienes y servicios médicos (BERGALLO, 2013; MOTTA FERRAZ, 2009; 2013; VENTURA et al., 2010), así como a promover una medicina defensiva (GHERARDI; GHERARDI, 2007). Respecto a este último punto, algunos autores apuntan el riesgo de que sea la justicia el ámbito en que se decidan los actos médicos.

Desde esta perspectiva, que terceras partes intervengan en este tipo de decisiones, que deberían tomarse en la intimidad de la relación médico-paciente, socava la confianza de los profesionales respecto a sus propias prácticas y distorsiona la relación terapéutica, generando prácticas iatrogénicas. La judicialización de casos en que no existe una disputa entre las partes (profesionales y pacientes y/o familiares), así como aquellos en que la legislación vigente o la jurisprudencia hacen superflua una autorización judicial, sustentan estas posiciones críticas. Muchos de los fallos del periodo son originados porque una de las partes recurre en forma preventiva a la justicia en busca de un aval judicial innecesario. Como señala Zussman (1992) a propósito de la limitación del esfuerzo terapéutico en unidades de terapia intensiva, a menudo existe una brecha entre lo que la ley efectivamente exige y lo que los profesionales interpretan o creen que exige, generando distorsiones y mayor burocracia en este tipo de decisiones. Asimismo, la sanción de normas o jurisprudencia relevante en determinado tópico no necesariamente impide la judicialización, como lo evidencia la litigación luego de la aprobación de la ley de muerte digna o incluso la presencia recurrente de demandas en torno al rechazo de transfusiones luego de fallos de la Corte Suprema de Justicia.

Otra lectura de la judicialización de la toma de decisiones en el final de la vida podría subrayar, sin embargo, que estos procesos suponen una mayor regulación de las atribuciones de los profesionales de la salud, tanto por la entrada en escena nuevos actores como por la instalación progresiva de la autonomía de los pacientes como valor central en estas decisiones. Los llamados a mantener en este ámbito las decisiones pueden encubrir la defensa de la discreción médica sobre estos asuntos, como lo muestra la literatura socio-antropológica sobre la gestión médica de la muerte y el morir (ver ALONSO, 2012) y las disputas entre profesionales y pacientes y/o familiares en varios de los casos analizados. 
Como señala Smulovitz (2008b), las prácticas de judicialización suponen la presencia de jueces y de tribunales como partes legítimas y autorizadas en la resolución de conflictos y disputas. Realizar o no una intervención quirúrgica, continuar o no un tratamiento, retrasar la agonía o permitir que acontezca la muerte eran hasta hace relativamente poco prerrogativa de los médicos, desde hace un tiempo discutida por abogados, jueces y los propios pacientes y familiares, como lo evidencia la evolución de los fallos en el periodo analizado. Estas transformaciones se inscriben en un proceso más amplio de regulación de la práctica y la investigación médicas por parte del Estado, en el que la emergencia y desarrollo de la bioética tiene un lugar destacado (ROTHMAN, 1991), y en la judicialización de diferentes aspectos de la vida y las relaciones sociales, anteriormente reguladas de manera informal y en el ámbito privado (SMULOVITZ, 2008; O’DONNELL, 2008).

La apelación a un discurso de derechos, sin embargo, no ha sido exclusivo de las estrategias de judicialización de los reclamos en esta materia, como se desprende de los debates que dieron pie a la aprobación de la ley de "muerte digna” en Argentina, forzados por la visibilidad de las demandas de pacientes y familiares, claves para el tratamiento parlamentario y la posterior concreción de iniciativas en este sentido (ALONSO et al., 2014). Estos casos, que evitaron adrede la estrategia individual de judicialización, dan cuenta sin embargo de la extensión y del potencial del discurso de los derechos, así como otras formas (más políticas) de reclamar en materia de derechos sobre el propio cuerpo y de reivindicar la autoridad sobre toma las decisiones en el final de la vida (ALONSO; PECHENY, en preparación). Tanto las demandas públicas por la sanción de una ley, como las demandas particulares encauzadas por vía judicial, dan cuenta de que la ciudadanía percibe en términos de derechos la toma de decisiones en situaciones extremas en las que está en juego la dignidad personal, y que es obligación del Estado garantizar el ejercicio pleno de la Rautonomía en esas instancias.

Como se ha evidenciado en las páginas anteriores, la sanción de leyes o jurisprudencia del máximo tribunal no limita el recurso a la justicia por parte de los actores involucrados, por lo que cabe preguntarse hacia dónde puede dirigirse la futura judicialización de las decisiones en el final de la vida. Al margen de posibles pedidos de eutanasia o suicidio asistido, no comprendidos en 
la legislación existente en Argentina, la emergencia de nuevas categorías clínicas y avances científicos pueden concentrar los futuros debates éticos y jurídicos, como los recientes descubrimientos en torno a los estados de conciencia mínima. En virtud de observaciones clínicas e imágenes cerebrales que parecían demostrar manifestaciones aisladas de conciencia entre pacientes en estado vegetativo (inconscientes por definición), en el año 2002 se definió esta nueva entidad clínica, que permite distinguir aquellos pacientes que pese a la gravedad de los daños neurológicos muestran signos evidentes de conciencia, aunque en forma lábil y fluctuante (DEHAENE, 2014; GIACINO et al., 2014). ${ }^{10}$ Los avances en estos campos, que podrían respaldar posiciones más cautelosas y/o conservadoras en la toma de decisiones en el final de la vida, no han encontrado aun sus voceros entre aquellos que se oponen a la suspensión de las medidas soporte vital en pacientes con alteraciones de conciencia, como algunos sectores de la Iglesia Católica y movimientos pro-vida.

\section{A modo de cierre}

En este artículo se abordó la judicialización de la toma de decisiones médicas en el final de la vida en Argentina, proceso enmarcado en la creciente judicialización de las relaciones sociales y de un mayor reconocimiento de la autonomía de los pacientes. A partir del análisis de un conjunto de fallos judiciales producidos a lo largo de cuatro décadas (1975-2015), se describieron el contexto y las principales características de la litigación respecto a estas decisiones, así como los cambios producidos en el período: la variación en los tipos de casos objeto de disputa (desde el rechazo de transfusiones al pedido de retiro de medidas de soporte vital), en los lenguajes y argumentos utilizados (de la objeción de conciencia a la autonomía y la dignidad), y la creación de nuevos derechos.

Asimismo, se ponderaron los alcances y efectos de la judicialización en este ámbito, como los aspectos negativos y positivos de estos procesos, se discutió el lugar del discurso de los derechos en los reclamos individuales y políticos respecto a la toma de decisiones en el final de la vida, y se perfilaron las alternativas posibles de la judicialización en este campo. Los avances científicos y su recepción por parte de la sociedad, y la radicalización de las demandas de los ciudadanos en relación a la autonomía (pedidos por la autorización de la 
eutanasia y el suicidio asistido), seguramente serán los ejes en torno a los cuales girarán los debates futuros.

Las decisiones en el final de la vida, tanto aquellas que se producen en forma cotidiana en ámbitos médicos como las que se gestan en instancias y procesos judiciales, ponen en juego ideas acerca de la dignidad personal y la libertad individual. La observación de los cambios que se producen en estas decisiones a lo largo del tiempo da cuenta del lugar central que van adquiriendo la autonomía personal y los intereses de los pacientes, y a la vez de los límites en la titularidad y el ejercicio de derechos sobre el propio cuerpo. Límites que remiten a aspectos formales del marco normativo en Argentina, como la penalización de la eutanasia y de cualquier forma de suicidio asistido, y a las condiciones institucionales en que estas decisiones tienen lugar, que previenen de sobreestimar el peso de la autonomía. En contextos médicos, la toma de decisiones en el final de la vida es producto de negociaciones entre actores con diferentes posicionamiento en las estructuras de poder y capacidad de agencia, dada la asimetría constitutiva de la relación médicopaciente, en los que las lógicas institucionales y determinadas prácticas (desde el lugar que los profesionales dan a los pacientes en estas decisiones a las formas en que pacientes y familiares son -o no- informados de la condición de salud y de las opciones terapéuticas) limitan los márgenes de acción y consentimiento.

Más allá del valor de describir el tratamiento de estas temáticas para el propio campo del derecho o disciplinas implicadas en estos procesos (como la bioética), las discusiones que se producen en la arena jurídica no pueden aislarse del contexto social más amplio en que estas instancias se inscriben. En este sentido las decisiones judiciales ponen de manifiesto formas en que se delinean socialmente los contornos de lo que se considera una "muerte digna" y el lugar que la tecnología médica adquiere en tales definiciones, así como las nociones de persona y los límites de la vida que en última instancia se discuten en casos como los expuestos en el análisis de los fallos.

Examinar la jurisprudencia sobre la toma de decisiones en el final de la vida permite iluminar así los discursos científicos y religiosos y los valores morales y comunitarios que configuran nuestras formas de interpretar la finitud y los límites de la existencia, así como las negociaciones, tensiones y acuerdos (contingentes y siempre revisables) acerca de las que consideramos vidas dignas de ser vividas y las formas aceptables de morir. 


\section{Agradecimientos}

La investigación se lleva a cabo con el apoyo del Consejo de Investigaciones Científicas y Técnicas (CONICET) y de la Agencia Nacional de Promoción Científica y Tecnológica de Argentina. Agradezco a uno de los evaluadores anónimos por los comentarios y sugerencias que enriquecieron el texto.

\section{Referencias}

ABDO FEREZ, C. La política y la juristocracia. Sociedad, n. 33, p. 51-72, 2014.

ALONSO, J. P. Contornos negociados del buen morir: la toma de decisiones médicas en el final de la vida”. Revista Interface - Com., Sau., Edu, v. 16, n. 40, p. 191-203, 2012.

. De medios ordinarios y extraordinarios. La Iglesia Católica y los debates sobre la “muerte digna” en Argentina. Religão e Sociedade, v. 34, n. 1, p. 122-145, 2014.

ALONSO, J. P. et al. El final de la vida como objeto de debate público: avatares de la "muerte digna” en Argentina". Revista Sociedad, n. 33, p. 7-20, 2014.

ALONSO, J. P.; PECHENY, M. (en preparación) Estrategias de judicialización y politización de reclamos relativos al cuerpo y la salud: el derecho a una "muerte digna".

ASENSI, F. Judicialização ou juridicização? As instituições jurídicas e suas estratégias na saúde. Physis, v. 20, n. 1, p. 33-55, 2010.

BERGALLO, P. Argentina: los tribunales y el derecho a la salud. ¿Se logra justicia a pesar de la "rutinización" de los reclamos individuales de cobertura? In: YAMÍN A., GLOPPEN, S. (Coord.) La lucha por los derechos de la salud. ¿Puede la justicia ser una herramienta de cambio? Buenos Aires: Siglo XXI, 2013. p. 59-95.

CARVALHO, E. R. Em busca da judicialização da política no Brasil: apontamentos para uma nova abordagem. Revista Sociologia Política, n. 23, p. 115-126, 2004.

CASTRA, M. Bien mourir. Sociologie des soins palliatifs. Paris: PUF, 2003. 367p.

CLARK, D.; SEYMOUR, J. Reflections on palliative care. Buckingham: Open University Press, 1999. 224p.

CONSTABLE, C. Withdrawal of artificial nutrition and hydration for patients in a permanent vegetative state: changing tack. Bioethics, v. 26, n. 3, p. 157-163, 2012.

CUENCA, A.; PICCONE, M. Nuevos movimientos sociales en Argentina y judicialización de demandas. Derecho y Ciencias Sociales, n. 5, p. 30-50, 2011.

DELAMATA, G. Amanecer y progreso de un repertorio social activista de derechos bajo la democracia recuperada. Revista SAAP, v. 7, n. 2, p. 307-315, 2013.

DEHAENE, S. La conciencia en el cerebro. Buenos Aires: Siglo XXI, 2015. 348 p. 
DINIZ, D. Quem autoriza o aborto seletivo no Brasil? Médicos, promotores e juízes em cena. Physis: Revista de Saúde Coletiva. Rio de Janeiro, v. 13, n. 2, p. 251-272, 2003.

- Quando a morte é um ato de cuidado: obstinação terapêutica em crianças. Cadernos de Saúde Pública, v. 22, n. 8, p. 1741-1748, 2006.

DINIZ, D.; MACHADO, T.; PENALVA, J. A judicialização da saúde no Distrito Federal, Brasil. Ciência \& Saúde Coletiva, v. 19, n. 2, p. 591-598, 2014.

FARJI-NEER, A. Producción generizada de los cuerpos en el discurso jurídico argentino. Análisis de tres fallos correspondientes al periodo 1970 - 2010. Equidady Sociedad, n. 3, p. 66-87, 2012.

FINS, J. Rights come to mind: brain injury, ethics and the struggle for consciousness. Cambridge: Cambridge University Press, 2015. 250 p.

FRIGERIO, A. "La invasión de las sectas": el debate sobre los nuevos movimientos religiosos en los medios de comunicación en Argentina. Sociedad y Religión, n. 10/11, p. 24-51, 1993. GHERARDI, C. Vida y muerte en terapia intensiva. Buenos Aires: Biblos, 2007. 180 p.

GHERARDI, C.; GHERARDI, N. La judicialización del acto médico y la generación de nuevos conflictos. Medicina (Buenos Aires), n. 67, p. 502-510, 2007.

GIACINO, J. et al. Disorders of consciousness after acquired brain injury: the state of the science. Nature Reviews Neurology, n. 10, p. 99-114, 2014.

GLOPPEN, S. La lucha por los derechos de la salud. Marco de análisis (pp. 31-55). In: YAMÍN, A.; GLOPPEN, S. (Coord.) La lucha por los derechos de la salud. ¿Puede la justicia ser una herramienta de cambio? Buenos Aires, Siglo XXI, 2013. p. 31-55.

HOOFT, P. Bioética y jurisprudencia. Acta Bioethica, n. 2, p. 211-237, 2002.

HOOFT, P.; PICARDI, G. Bioética y derecho. El consentimiento informado en la Jurisprudencia Argentina. Revista Latinoamericana de Bioética, v. 10, p. 1-58, 2006.

LOCK, M. Twice dead: organ transplants and the reinvention of death. Berkeley: University of California Press, 2002. 441p.

MCIRENEY, F. "Request death": a new social movement. Social Science \& Medicine, v. 50, p. $137-154,2000$.

MENEZES, R. "Difíceis decisões: uma abordagem antropológica da prática médica em CTI”. Physis; Revista de Saúde Coletiva. Rio de Janeiro, v. 10, n. 2, p. 27-49, 2000.

. Em busca da boa morte: antropologia dos cuidados paliativos. Río de Janeiro: Garamond/Fiocruz, 2004. 228p.

MOTTA FERRAZ, O. L. The right to health in the courts of Brazil: worsening health inequities? Health and Human Rights, v. 11, n. 2, p. 33-45, 2009. 
NETTELTON, S.; KITZINGER, J.; KITZINGER, C. A diagnostic illusory? The case of distinguishing between "vegetative" and "minimally conscious" states. Social Sciences \& Medicine, n. 116, p. 134-141, 2014.

ROTHMAN, D. Strangers at the bedside.New York: Basic Books, 1991. 320 p.

SIEDER, R.; SCHJOLDEN, L.; ANGALL, A., (Ed.) La judicialización de la politica en América Latina. Bogotá: Universidad Externado de Colombia, 2008. 338 p.

SMUlOVITZ, C. Petición y creación de derechos: la judicialización en Argentina. En: SIEDER, R.; SCHJOLDEN, L.; ANGALL, A. (Eds.). La judicialización de la política en América Latina. Bogotá: Universidad Externado de Colombia, 2008. p. 193-221.

Organizaciones que invocan derechos. Sociedad civil y representación en Argentina. POSTData, v. 13, p. 51-79, 2008b.

VENTURA, M. et al. Judicialização da saúde, acesso à justiça e efetividade do direito à saúde. Physis: Revista de Saúde Coletiva. Rio de Janeiro, v. 20, n. 1, p. 77-100, 2010.

VENTURA, M. A transexualidade no tribunal: saúde e cidadania. Río de Janeiro: EdUERJ, 2010.

YAMÍN, A.; GLOPPEN, S. (Coord.) La lucha por los derechos de la salud. ¿Puede la justicia ser una herramienta de cambio? Buenos Aires: Siglo XXI, 2013. 488p.

ZUSSMAN, R. Intensive care. Medical ethics and the medical profession. Chicago: University of Chicago Press, 1992. 260 p.

\section{Notas}

${ }^{1}$ Datos del proyecto y del financiamiento.

${ }^{2}$ Se analiza un corpus conformado por 38 fallos, relevados en cinco bases de datos jurídicas: los portales La Ley online e Infojus, y las bases virtuales de la Corte Suprema de Justicia de la Nación Argentina, de la Suprema Corte de la Provincia de Buenos Aires y del Tribunal Superior de Justicia de la Ciudad Autónoma de Buenos Aires. La búsqueda fue realizada a partir de descriptores ("muerte digna”, "eutanasia", "objeción de conciencia”, entre otros), y complementada con un trabajo de archivo. El texto de los fallos fue analizado a partir de técnicas cualitativas por medio del programa Altas.ti.

${ }^{3}$ El inciso 3 del artículo 19 de la ley 17.132 señala que entre las obligaciones de los profesionales está la de: "respetar la voluntad del paciente en cuanto sea negativa a tratarse o internarse, salvo los casos de inconsciencia, alienación mental, lesionados graves por causa de accidentes, tentativas de suicidio o de delitos. En las operaciones mutilantes se solicitará la conformidad por escrito del enfermo, salvo cuando la inconsciencia o alienación o la gravedad del caso no admitiera dilaciones. En los casos de incapacidad, los profesionales requerirán la conformidad del representante del incapaz".

${ }^{4}$ Analizando la emergencia de nuevos movimientos religiosos en Argentina, Frigerio (1993) señala la preocupación social generada por la actividad de estos grupos, considerados como sectas, como los sacrificios atribuidos a la religión Umbanda y el rechazo a las transfusiones sanguíneas por parte de los Testigos de Jehová, en especial cuando involucran a menores. Si bien a menudo se incluyen en la 
caracterización de los nuevos movimientos religiosos en la región, los Testigos de Jehová han suscitado un menor interés por parte de la academia en comparación a otros grupos. Los estudios que centran el análisis en este grupo suelen abordar la temática de las transfusiones desde una perspectiva bioética y médica, y no tanto desde los abordajes más clásicos de la sociología de la religión.

${ }^{5}$ En Argentina, como señala Bergallo (2013), nuevas herramientas procesales y la suscripción de tratados de derechos humanos que reconocen en forma explícita el derecho a la salud, incorporados por la reforma constitucional de 1994, dinamizaron de manera notoria los procesos de judicialización en el campo de la salud.

${ }^{6} \mathrm{El}$ soporte vital "comprende todos los procedimientos asistenciales (instrumentales, electrónicos o farmacológicos) que sustituyen o apoyan las funciones de órganos o sistemas cuya afectación pone en peligro la vida” (GHERARDI, 2007, p. 36), desde aquellos más sofisticados, como los respiradores mecánicos, a los de menor complejidad, como la nutrición e hidratación.

${ }^{7}$ El estado vegetativo supone un daño en la corteza cerebral que ocasiona la pérdida de la conciencia (la atención, la memoria y la comunicación), aunque el cuerpo conserve reflejos y movimientos espontáneos. La prolongación de esta condición en el tiempo deriva en el "estado vegetativo permanente", que supone la irreversibilidad del daño (GHERARDI, 2007).

${ }^{8}$ La judicialización preventiva que ilustra este tipo de casos ha sido criticada por aquellos que señalan que el respeto de estas decisiones deben quedar en el marco de una relación terapéutica, máxime cuando no existen conflictos entre las posiciones de los profesionales y los pacientes y/o sus familiares (ver GHERARDI; GHERARDI, 2007).

9 En Argentina existe un "control de constitucionalidad difuso", que "habilita a todos los jueces a pronunciarse sobre la constitucionalidad de una norma" (GHERARDI; GHERARDI, 2007, p. 506; HOOFT, 2002); uno de los medios en que se expresa la judicialización de la política y la intervención de los jueces en las políticas públicas (SIEDER et al., 2008).

${ }^{10}$ Investigaciones recientes demostraron que estos pacientes conservan la capacidad de percibir dolor y de procesar el lenguaje. Para una revisión de los avances de la investigación neurocientífica sobre los desórdenes de conciencia ver Giacino et al. (2014) y Dehane (2014). 
O direito a uma morte digna na Argentina: a judicialização da tomada de decisóes médicas no final da vida

$\mathrm{O}$ artigo discute, a partir de uma perspectiva sócio-histórica, o contexto e implicaçōes sociais da judicialização da gestão médica da morte e o morrer. O objetivo do artigo é descrever e analisar a judicialização da tomada de decisōes médicas no final da vida na Argentina, a partir de julgamentos produzidos entre 1975 e 2015. O artigo examina o contexto e as principais características da litigação em torno a essas decisōes, assim como as mudanças no período: nos casos disputados (de rejeitar transfusōes a pedidos de retirada de suporte vital), em os argumentos e linguajem usados (de objeção de consciência à autonomia) e o surgimento de novos direitos. Por fim, são discutidas as consequências e perspectivas futuras da judicialização neste campo.

> Palavras-chave: judicialização; medicalização; morte digna; bioética; Argentina. 
The right to a dignified death in Argentina: the judicialization of end-of-life care decisions The paper discusses, from a socio-historical perspective, the context and implications of judicialization processes regarding medical decisions making on death and dying. This paper aims to describe and analyze the judicialization of end-of-life care decisions at the end of life in Argentina, considering courts decisions produced between 1975 and 2015. The context and the main features of litigation regarding those decisions are examined, as well as the changes within the period: the cases disputed (from rejecting blood transfusions to withdrawal of life support), the arguments and languages that frame the legal cases (form conscientious objection to autonomy) and the emergence of new rights. Finally, the social consequences and the future prospects of judicialization in this field are discussed.

Key words: judicialization; medicalization; death with dignity; bioethics; Argentina. 